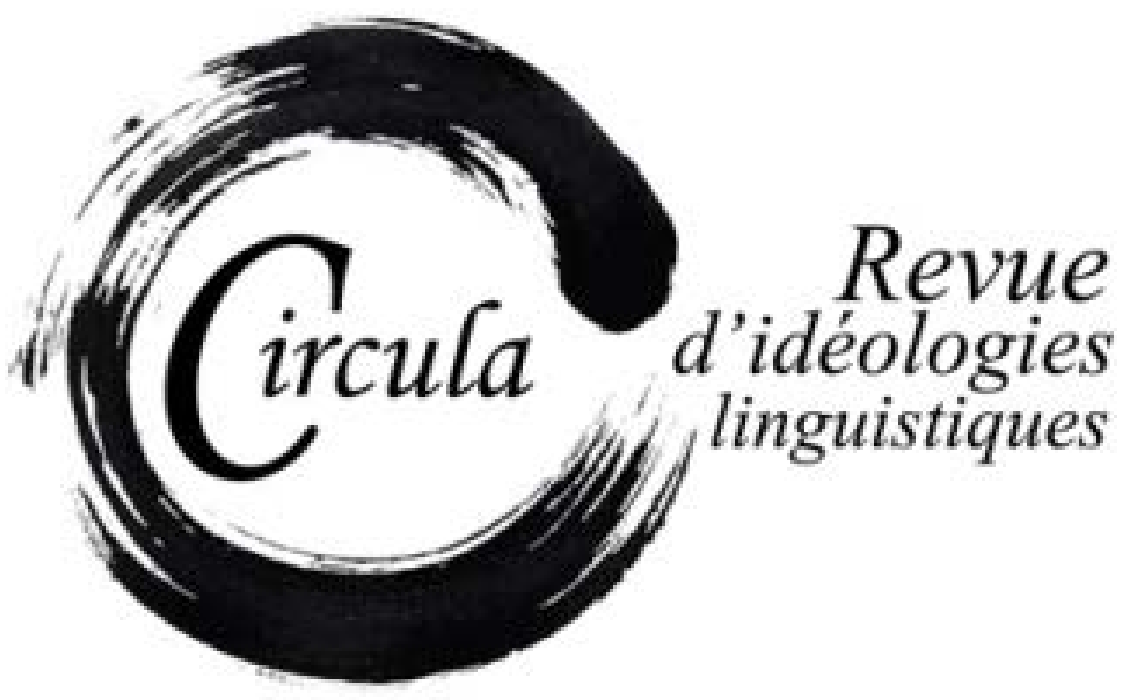

TITRE: DALLA QUESTIONE DELLA LINGUA ALL'AGGRESSIONE LINGUISTICA: LE IDEE SULLA LINGUA NEI GIORNALI ITALIANI DELL'ULTIMO DECENNIO

Auteur(s): FABIO RossI, UnIVERSITÀ DI MESSINA

Revue: Circula, NUmÉRO 1, PAGES 173-95

ISSN: 2369-6761

DiRECTEURS: Wim REMYSEN ET SABINE SCHWARZE

URI: HTTP://HDL.HANDLE.NET/11143/8002

DOI: $10.17118 / 11143 / 8002$ 


\section{Dalla questione della lingua all'aggressione linguistica: le idee sulla lingua nei giornali italiani dell'ultimo decennio}

Fabio Rossi, Università di Messina fab.ros67@alice.it

Riassunto: L'articolo è suddiviso in tre parti. Nella prima, dall'esame di brani dalla Repubblica e dal Corriere della sera si osserva in che modo i giornali trattano questioni linguistiche. Nella seconda, si esemplifica il fenomeno della discriminazione mediante l'analisi di alcuni etnonimi (siciliano, calabrese, milanese, torinese, ecc.). Nella terza parte, si analizza un interessante strumento lessicografico del settimanale L'Espresso: Slangopedia, sui gerghi giovanili. A tenere insieme questi tre temi è il rapporto tra media e ideologia linguistica, nell'intento di stimolare la ricerca futura a investigare sull'identità linguistica degli italiani, attraverso la stampa periodica, come riflesso dell'identità culturale. Se ne ricava che la società italiana non sembra più in grado di riflettere criticamente sulla propria lingua: in molti articoli, infatti, spicca il tono aggressivo e forse discriminatorio nei confronti di chi si allontana da uno standard spesso male interpretato.

Parole chiave: giornali; italiano standard; variabilità; dialetto; discriminazione; gergo; identità

Abstract: This paper is divided into three sections. In the first one, some articles from la Repubblica and Corriere della sera will be analyzed to show how newspapers deal with language issues. In the second section, the phenomenon of discrimination will be illustrated through the analysis of some ethnonyms (siciliano, calabrese, milanese, torinese and so on). In the third section, an interesting lexicographical tool in the magazine L'Espresso will be discussed: Slangopedia, a dictionary of Italian slangs. The relationship between mass media and linguistic ideology brings together these three sections and aims to encourage future research to investigate, through the newspapers, the Italian linguistic identity as a reflection of the Italian cultural identity. In the conclusive remarks, it will be shown that the Italian society no longer seems able to critically reflect on its own language: in many articles, in fact, an aggressive and discriminatory tone is unleashed against those who do not use the linguistic standard.

Keywords: newspapers; standard Italian; variation; dialect; discrimination; slang; identity 


\section{Introduzione ${ }^{1}$}

Il presente contributo è strutturato in tre parti. Nella prima, si darà conto del ruolo dei giornali nel trattare questioni relative alla lingua italiana e agli italiani regionali. Nella seconda, si esemplificherà il fenomeno della discriminazione mediante l'analisi degli etnonimi utilizzati dai giornali. Nella terza parte, infine, si analizzerà un interessante strumento lessicografico collegato al settimanale L'Espresso. A tenere insieme questi tre temi è il rapporto tra media e ideologia linguistica, nella convinzione che quest'ultima possa reperirsi non soltanto nelle riflessioni dei grammatici e dei linguisti di professione, ma anche nelle considerazioni dei giornalisti e dei lettori (nel senso, ormai consolidato, dunque, della cosiddetta linguistica popolare o laica: cf. Sgroi, 2010) e che faccia parte integrante, pertanto, del più ampio quadro dell'identità culturale di una società.

La natura volutamente eterogenea dei tre temi qui esposti si deve all'intento di questo articolo di porsi come stimolo alla ricerca futura a investigare su simili argomenti, piuttosto che come punto di arrivo su dati ancora in via di elaborazione e oggetto di riflessione.

Nella prima e nella seconda parte del lavoro, ho attinto ad articoli dai due principali quotidiani italiani (scelti proprio in quanto rappresentativi, in virtù dell'ampia tiratura, della temperie culturale della Penisola): la Repubblica e Corriere della sera, con particolare riferimento agli anni 2012 e 2013.

Ho preferito concentrarmi su raccolte di dati di prima mano e sul commento degli articoli più significativi tra quelli individuati, adottando quindi un'ottica nettamente sbilanciata su aspetti pratici (e basati su corpora costruiti ad hoc e dunque analizzando un campione limitato di dati), piuttosto che di inquadramento teorico, per due motivi:

1. Il tema dell'identità e dell'ideologia linguistica veicolate dai media, assai florido e maturo all'estero, è in Italia praticamente sconosciuto. Per questo motivo, ritengo che un'attenta riflessione teorica debba forse ancora attendere e seguire un ampio lavoro di raccolta di dati.

2. Utili riferimenti metodologici e inquadramenti teorici sul tema dell'ideologia linguistica trattato fuori d'Italia possono essere reperiti in molti dei contributi raccolti in questa stessa sede; un rinvio ad essi sarà più utile di una ripetizione.

Più che a conclusioni certe, pertanto, il presente studio condurrà alla formulazione di molte domande e ipotesi, con lo scopo di incentivare approfondimenti futuri su questi temi. In quest'ottica andrà inteso l'andamento talora discorsivo e interlocutorio, piuttosto che deduttivo e analitico, delle riflessioni che seguono.

1. Sono grato a Maria Grazia Sindoni per i suggerimenti. Avverto che tutti i siti internet citati nel presente articolo sono stati regolarmente consultati fino al 10 febbraio 2014. 
Partiamo da una doppia citazione dal Quaderno 29 (l'ultimo, del 1935: per la datazione dei quaderni gramsciani cf. Mordenti, 1996: 581; su Gramsci, e in genere sulle ideologie linguistiche del primo Novecento, cf. ora Demartini, 2014: 181-184 et passim) di Antonio Gramsci, che ci accompagna direttamente dentro la questione dell'ideologia linguistica, ovvero la questione italiana della lingua:

Manzoniani e «classicisti». Avevano un tipo di lingua da far prevalere. Non è giusto dire che queste discussioni siano state inutili e non abbiano lasciato tracce nella cultura moderna, anche se non molto grandi. In realtà in questo ultimo secolo la cultura unitaria si è estesa e quindi anche una lingua unitaria comune. Ma tutta la formazione storica della nazione italiana era a ritmo troppo lento. Ogni volta che affiora, in un modo o nell'altro, la quistione della lingua, significa che si sta imponendo una serie di altri problemi: la formazione e l'allargamento della classe dirigente, la necessità di stabilire rapporti piú intimi e sicuri tra i gruppi dirigenti e la massa popolare-nazionale, cioè di riorganizzare l'egemonia culturale. Oggi si sono verificati diversi fenomeni che indicano una rinascita di tali quistioni: pubblicazioni del Panzini, Trabalza-Allodoli, Monelli, rubriche nei giornali, intervento delle direzioni sindacali, ecc. (Gramsci, 1996: 129)

Poco sotto Gramsci scriveva:

La cosí detta «quistione della lingua». Pare chiaro che il De Vulgari Eloquio di Dante sia da considerare come essenzialmente un atto di politica culturale-nazionale (nel senso che nazionale aveva in quel tempo e in Dante), come un aspetto della lotta politica è stata sempre quella che viene chiamata «la quistione della lingua» che da questo punto di vista diventa interessante da studiare. Essa è stata una reazione degli intellettuali allo sfacelo dell'unità politica che esistè in Italia sotto il nome di «equilibrio degli Stati italiani», allo sfacelo e alla disintegrazione delle classi economiche e politiche che si erano venute formando dopo il Mille coi Comuni e rappresenta il tentativo, che in parte notevole può dirsi riuscito, di conservare e anzi di rafforzare un ceto intellettuale unitario, la cui esistenza doveva avere non piccolo significato nel Settecento e Ottocento (nel Risorgimento). Il libretto di Dante ha anch'esso non piccolo significato per il tempo in cui fu scritto; non solo di fatto, ma elevando il fatto a teoria, gli intellettuali italiani del periodo piú rigoglioso dei Comuni, «rompono» col latino e giustificano il volgare, esaltandolo contro il «mandarinismo» latineggiante, nello stesso tempo in cui il volgare ha cosí grandi manifestazioni artistiche. Che il tentativo di Dante abbia avuto enorme importanza innovatrice, si vede piú tardi col ritorno del latino a lingua delle persone colte (e qui può innestarsi la quistione del doppio aspetto dell'Umanesimo e del Rinascimento, che furono essenzialmente reazionari dal punto di vista nazionale-popolare e progressivi come espressione dello sviluppo culturale dei gruppi intellettuali italiani e europei). (Gramsci, 1996: 131)

I dibattiti intorno a una lingua, dunque, specialmente se assumono la forma dei trattati (o anche solo delle grammatiche e dei dizionari), hanno, secondo Gramsci, una forte natura ideologica e politica, in 
quanto i concetti di unità/varietà, norma/uso, comunicazione pubblica/privata, lingua di un popolo/ lingue di altri popoli non possono che legarsi ai più profondi concetti di identità/alterità, spirito nazionale, rapporti di potere tra classi sociali, ecc.

\section{Il dibattito linguistico nei quotidiani italiani}

Allontanandoci per ora dagli scrittoi dei linguisti, che cosa succede nei massimi quotidiani italiani oggi, a proposito dei dibattiti linguistici? Diciamo subito che emerge con chiarezza una certa inversione di tendenza, rispetto ai decenni scorsi. Certo, è difficile portare evidenze numeriche. Per gli anni 1962-63 e 1972-73 siamo abbastanza fortunati, poiché disponiamo dello spoglio di Carrafiello $(1977)^{2}$, dal quale si ricava una notevole mole di articoli, nella principale stampa quotidiana italiana dell'epoca, d'argomento linguistico, attorno ai seguenti temi principali: forestierismi (soprattutto l'inglese) e dialetti (soprattutto il napoletano). Seguono poi, più o meno in ordine decrescente e molto meno frequenti, i seguenti temi: nomi (forma, significato, ecc.), lingue speciali e gerghi, didattica dell'italiano, neologismi, pronuncia (radiotelevisiva), televisione e radio, verbi, aggettivi, accenti, cinema, ecc.

Abbastanza numerose sono state, nel passato, le rubriche linguistiche nei vari quotidiani (di talune si parla anche in questa sede), che invece, oggi, sembrano ridotte a due soli casi notevoli: Lessico e nuvole di Stefano Bartezzaghi (in forma solo scritta), nella Repubblica (dal $1^{\circ}$ febbraio 2013), tuttora in corso, e 3 minuti 1 parola (in forma audiovisiva) di Beppe Severgnini, nel Corriere della sera online (dal 31 gennaio 2011), conclusa il 13 giugno 2013, alla centesima puntata.

Un dibattito linguistico largamente seguito dalla stampa nell'ultimo decennio è stato quello attorno alla proposta di istituzione di un Consiglio Superiore della Lingua Italiana, dapprima nel 2001, ad opera del senatore di Forza Italia Andrea Pastore. Del caso, poi naufragato nel nulla, si è continuato a parlare con un certo fervore almeno fino al 2005 (cf. Arcangeli, 2004 ed altri interventi dedicati al medesimo tema nello stesso I numero della collana LId'O; cf. anche Serianni, 2005). L'ampia letteratura critica in merito alla questione ci solleva dal compito di riassumerla minutamente, se non per ricordare che, tra i compiti del Consiglio Superiore, avrebbe dovuto esserci anche l'«elaborazione di una grammatica "ufficiale" della lingua italiana» e la «compilazione di un dizionario dell'“uso", da mantenere in costante aggiornamento» (Serianni, 2005: 60), compiti poi espunti dal testo della nuova proposta.

Da allora, di problemi legati alla grammatica, alla questione della lingua (in fondo mai sopita, in Italia) e soprattutto ai dubbi e alle proteste degli italiani che la parlano e la scrivono si continua a dibattere, moltissimo, in sedi più o meno specialistiche (dalla collana LId'O, diretta da Massimo Ar-

2. Le storiche polemiche (di Pasolini, Calvino e altri) intorno alla cosiddetta «nuova questione della lingua» italiana, fiorite perlopiù negli anni sessanta del Novecento e di grande circolazione mediatica all'epoca, possono essere lette in Parlangeli (1971). 
cangeli per Bulzoni, al foglio La Crusca pervoi, dell'omonima Accademia fiorentina) e in rete: dal blog Il linguista della Repubblica online ${ }^{3}$, a migliaia di dibattiti tra naviganti ${ }^{4}$, per arrivare a veri e propri siti professionali, da quello della Treccani ${ }^{5}$ a quello di Carocci $^{6}$, animato, tra gli altri, da chi scrive. Per menzionare altri media, si può ricordare la trasmissione La lingua batte, di Giuseppe Antonelli, su Rai Radio 3. Anche l'editoria legata a pubblicazioni di grammatica spicciola, per dir così, è sempre più florida, segno di notevole interesse da parte del grande pubblico. Eppure di questi temi si parla molto di meno, oggi rispetto a ieri, nei quotidiani e nei settimanali nazionali, nei quali, a ben guardare, quasi tutte le volte che i concetti, e le relative parole semplici o complesse, di lingua italiana, dialetto, grammatica o simili vengono evocati, lo si fa perlopiù a scopo denigratorio, aggressivo o demolitorio dell'altro, avallando peraltro triti, quanto spesso imprecisi, stereotipi (su denigrazione e stereotipi cf. Pistolesi, 2007, 2008; Rossi, 2011 e Redattore Sociale, 2013). Ė come se, insomma, i giornali italiani avessero consegnato ad altri media la funzione di intervenire, in modo un po' più approfondito, sulle questioni linguistiche. Perché? Un normale segno dei tempi? Ma vediamo prima di tutto qualche esempio di quella che ho definito aggressione linguistica, tratto da articoli raccolti tra la primavera 2012 e l'autunno 2013 nella Repubblica e nel Corriere.

\section{Cominciamo con un articolo della Repubblica del 12 settembre 2012:}

(1) Un continuo botta e risposta. Nel mirino dei leghisti è finito il deputato Udc Angelo Cera che, dopo un intervento molto duro contro i leghisti concluso con l'invito a lavarsi il muso prima di parlare del Sud, è stato fatto oggetto di attacchi che insistono soprattutto sulla necessità che il parlamentare pugliese faccia un bel corso di dizione e impari la lingua italiana prima di intervenire in aula. Il tutto condito da insulti che sono volati da un banco all'altro, dal classico «terroni» all'altrettanto classico «polentoni». Meno classico il «siete la Magna Grecia» nel senso che state «a magnà» le risorse del paese, sempre di matrice leghista.

3. http://linguista.blogautore.repubblica.it/

4. A partire da http://it.answers.yahoo.com.

5. http://ww.treccani.it

6. http://www.scrivereinitaliano.it. Si veda anche il neonato (marzo 2015) DICO - Dubbi sull'Italiano Consulenza Online, creato e amministrato da chi scrive e da Fabio Ruggiano in seno all'Università di Messina (e dunque di fatto il primo sito linguistico agganciato ad un'università italiana): http://www.unime.it/dico.

7. Tutti gli articoli di seguito citati si intendono prelevati dalle pagine online di repubblica.it e corriere.it. Quando questo saggio era già in bozza, è stato pubblicato Aresti (2014), utile rassegna di articoli d'argomento linguistico nella Repubblica e nel Corriere della sera dal 2009 al giugno 2013. Da una rapida scorsa agli argomenti e ai toni di questo materiale, mi pare emergano scarse variazioni sui soliti temi, più o meno superficialmente trattati: dannosa influenza dell'inglese e dei nuovi media, morte del congiuntivo, strumentalizzazione, in chiave leghistica, dei dialetti. Recentissimo (febbraio 2015) è anche il notevole interesse mediatico suscitato dall'iniziativa, di Annamaria Testa, «Dillo in italiano», contro l'abuso di anglicismi nella lingua (soprattutto politica) italiana: tutti i dettagli dell'iniziativa e i collegamenti ai numerosi articoli giornalistici ad essa legati si possono leggere in nuovoeutile.it. Chissà che quest'inedito interesse dei giornali a un simile tema non inauguri un'inversione di tendenza rispetto alla generale indifferenza della stampa attuale alle questioni linguistiche. 
Ecco ora invece un articolo del Corriere della sera del 20 dicembre 2012:

(2) Ma lì De Martino si è arrabbiato e ha cominciato a dirmi che non potevo rivolgermi alla sua collega in questo modo e, dopo essere scivolato su un congiuntivo, mi ha detto che gli facevo perdere anche la correttezza della lingua italiana. Io non avevo fatto niente, stavo solo spiegando cose che già dissi alla dottoressa Pagano.

Ho riportato non a caso due brani l'uno sulla pronuncia (o dizione) l'altro sul congiuntivo, perché pressoché tutti gli articoli d'ambito linguistico da me raccolti in Repubblica e nel Corriere tra il 2012 e il 2013 ruotano attorno a questi due stereotipi (la morte del congiuntivo e l'imbarbarimento della fonetica italiana a causa dei dialetti o, più raramente, delle lingue straniere), utilizzati quasi sempre per delegittimare un avversario perlopiù politico (della pletora di riferimenti sul congiuntivo, basti ricordare Della Valle e Patota, 2009 e Sgroi, 2013).

Un altro paio di esempi su temi analoghi. Corriere della sera del 4 luglio 2012:

(3) Egregio Direttore, pur di dipingermi nei panni del Fantozzi che sbaglia i congiuntivi, il Corriere della Sera on line adotta disinvoltamente il linguaggio insensato del Fracchia. Non ha infatti alcun significato la frase che il quotidiano on line mette assieme allo scopo di accreditare il mio presunto errore di congiuntivo. In tutta evidenza, non ho inteso pronunciare alcun «segui», bensì un «segni», (verbo segnare): leggendo il testo degli appunti scritto a mano, la n, come a volte accade, è stata scambiata per una u. Ne risulta che la frase, mondata dalle interpretazioni fracchiane, sia semplicemente e coerentemente questa: «Tuttavia non ci precludiamo la speranza che l'esito del vertice europeo segNi l'atteso cambio di rotta...». E' evidente che il segua, o il «segui» attribuitomi, non avrebbe avuto alcun senso: l'esito di un vertice, infatti, non può seguire alcunché, semmai è da questo risultato che possono prodursi delle conseguenze. Non credevo di avere alle mie spalle tanto solerti «correttori», fulminei nel cogliere ogni incertezza di dizione per trasformarla in orrori grammaticali. Ammetto, tuttavia, di non meritarmi la simpatia dei media, dopo la mia presa di posizione contro i finanziamenti di denaro pubblico alla carta stampata. Mi fa specie, tuttavia, che a darmi lezioni di grammatica siano i membri di un ordine professionale che, a differenza di quello a cui ho l'onore di appartenere, non richiede neppure il possesso della laurea come titolo minimo e necessario ai fini dell'iscrizione. D'altro canto, comprendo le necessità della stampa di creare e rinverdire lo stereotipo del padano ignorante. Io stesso vivo ancora di rendita per una circostanza attribuitami da anni a prova provata di asineria: quel «sarò breve e circonciso» che dissi all'indomani della mia elezione a sindaco di Lecco. Confesso: non di mio strafalcione si trattò, bensì della felice espressione pronunciata, molto tempo prima, da Diego Abatantuono nel suo «Eccezziunale veramente». Pensavo che la battuta e l'espressione scherzosa risultassero evidenti: ma a qualcuno, le cose vanno sempre spiegate a posteriori. 
Corriere della sera del 25 giugno 2013:

(4) Rivolgendosi a Cécile Kyenge, il parlamentare leghista ha alluso al fatto che la ministra all'Integrazione «ogni tanto fa torto alla lingua dei nostri padri». Senza rendersi evidentemente conto di aver appena commesso un torto linguistico ben più grave, affermando fra l'altro che "chi in questo Paese ci è nato e ci nascerà», dove quei due «ci» sarebbero stati sottolineati con la matita blu da qualunque insegnante di grammatica italiana. Ma se si dovesse star dietro alle improprietà espressive (e non solo grammaticali) dei politici aderenti al Carroccio si rischierebbe di far notte.

I due articoli appena citati, pur nella diversa tipologia sotto avvertita, sono interessanti anche per altri motivi. II primo dimostra quale importanza sia attribuita dai media agli errori di lingua, se il politico di turno (per la cronaca, il senatore leghista Lorenzo Bodega) risponde con un'aggressività pari a quella subita, per scongiurare, evidentemente, la sanzione sociale che gli italiani riservano a chi si macchia del reato di lesa maestà del congiuntivo.

Il secondo articolo, di là dalla comprensibile deprecazione del razzismo del deputato di turno (per la cronaca, l'onorevole leghista Massimo Bitonci), svela invece l'eccessivo purismo del giornalista: a ben guardare, a parte la scusabilissima dislocazione a sinistra, l'enunciato «chi in questo Paese ci è nato e ci nascerà» non sembra certo da stigmatizzare (se non nel contenuto).

Naturalmente sono ben conscio del fatto che, in un'eventuale continuazione di questa ricerca, sarebbe opportuno distinguere tra diversi tipi testuali: l'articolo scritto da un giornalista è differente dalla lettera al giornale, come il primo è diverso dalla cronaca linguistica (di cui peraltro in questa sede non mi occupo) del linguista di professione. Tuttavia, se lo scopo è, nel presente contributo, quello di concentrarsi sull'ideologia linguistica attraverso interventi di linguistica laica, credo sia legittimo, almeno preliminarmente, mettere sullo stesso piano i diversi articoli, indipendentemente dagli autori che li hanno scritti.

A volte l'accusa di abusare della grammatica e della lingua italiana è totalmente generica e priva di esempi, usata soltanto come arma di delegittimazione dell'altro:

(5) Sui provini del Grande Fratello, Matteo Garrone ci ha pure fatto un film, Reality, vincitore del Grand Prix al festival di Cannes. Come dargli torto: da Nord a Sud, un nugolo di ragazze scosciate, sedicenti studenti universitari, trombeur de femme o presunti tali, toraci depilati, padri di famiglia annoiati, macchiette di ogni tipo, ma soprattutto gente che la grammatica non sa manco cosa sia. (corriere.it, 6 giugno 2012) 
Talora, l'accusa di cattiva conoscenza dell'italiano sfuma in osservazioni velatamente razzistiche:

(6) Ore 23.05: risponde un signore decisamente gentile e decisamente ignaro della lingua italiana (segno inequivocabile della collocazione del call center, come d' uso, da qualche parte fra Tirana e Dushanbe). (repubblica.it, 12 agosto 2012)

Come già detto, non è facile, in questi casi, dare evidenze numeriche del fenomeno. Tuttavia, si può rilevare che, in 50 occorrenze del sintagma «lingua italiana» in repubblica.it, procedendo cronologicamente a ritroso dal $1^{\circ}$ ottobre 2013, 4 sono di chiara natura polemica o aggressiva. Se si considera che la gran parte delle occorrenze è non marcata in quanto totalmente referenziale (come quando si pubblicizzano corsi in lingua italiana, o indicazioni in lingua italiana), l'incidenza del fenomeno è di un certo rilievo. Lo stesso calcolo, per lo stesso periodo, fatto nel corriere.it porta al numero di 3 occorrenze. Riportiamo soltanto un caso, dei sette raccolti, da Repubblica del 18 agosto 2013, che mostra un Beppe Grillo scatenato contro gli eufemismi del politicamente corretto (evidentemente, da lui erroneamente scambiato col «parlare civile»: cf. Redattore sociale 2010):

(7) Chi dovesse esclamare, magari a pieno diritto, «paese di merda» sarebbe sanzionato, ma se avesse detto «Questo è il Paese che amo» sarebbe un perfetto candidato per la presidenza del Consiglio. Mentre parli devi continuamente e seriamente valutare se ogni parola che stai per pronunciare può urtare la sensibilità di qualcuno: un gruppo religioso, un'istituzione, una comunità, un'inclinazione sessuale, un'infermità, un popolo. Per non avere problemi devi limitarti ai saluti «Buongiorno e non mi faccia dire altro». Un immigrato clandestino è un rifugiato alla luce del sole. Razzi non è ignorante, non ha una perfetta padronanza della lingua italiana.

\section{Unitarietà contro molteplicità}

Stando al tono di questi articoli, si pretenderebbe spesso che la lingua fosse più unitaria, quasi monolitica (come avrebbe voluto il Consiglio Superiore della Lingua Italiana, con i suoi grammatica e dizionario ufficiali), di quanto non sia e non possa mai essere. I giornalisti (e i non linguisti in genere), molto spesso, sembrano non rendersi conto che l'unitarietà (geografica e sociale), in fatto di lingua, è sempre un valore relativo e negoziabile, e che il confine tra norma (pure esistente e codificata nelle grammatiche) e uso è spesso, e soprattutto oggi, più fluido di quanto non si creda. Ce lo ricorda Giovanni Nencioni, in un suo memorabile intervento del 1984:

Si sa bene in effetti che l'Italia, anziché di una lingua nazionale non solo unica ma unitaria, come il francese, l'inglese e il russo, dispone tuttora di una complessa mescolanza di lingua letteraria e dialetto, in cui si possono individuare sottounità regionali e una pluralità di registri considerabili come varianti sociostilistiche della medesima lingua; e che la stessa lingua letteraria è piuttosto un insieme di scelte stilistiche che una unità organica, un insieme entro il quale le grammatiche puristiche cercavano di isolare un nucleo privilegiato, producendo 
una strutturazione riflessa ed elitaria che solo mediatamente e lentamente poteva condurre ad una unificazione di ampiezza nazionale. La stessa forte spinta verso un italiano medio scritto e parlato, e veramente comune, venuta negli anni recenti dalla comunicazione di massa, pur producendo una progressiva riduzione delle varietà areali e il progressivo aumento delle isoglosse lessicali e morfosintattiche panitaliane (di quelle fonetiche non c'è per ora da fare conto), e quindi l'appiattimento dello spessore diacronico della lingua dei giovani, non vale ancora a conseguire quel livellamento sincronico che presentano il francese e l'inglese correnti. A tutt'oggi rendersi consapevoli della realtà linguistica italiana non si può senza fare della storia, né si può affrontare il problema di una norma valida non più per un ristretto gruppo d'italiani, ma per tutta la società italiana che parla e scrive, senza cogliere la diacronia che fermenta nella eterogenea sincronia dell'italiano odierno e ne intride i processi dinamici. (Nencioni, 1989: 223-224)

Il tono aggressivo, demolitorio dell'altro e cinico degli articoli di giornale sopra menzionati, ben lungi da qualunque pacato dibattito linguistico, o politico, non può non ricordare le parole che Giacomo Leopardi, come non mai profetico, spese nel suo Discorso sopra lo stato presente dei costumi degl'Italiani, nel 1824, a proposito del cinismo degli italiani: «Le classi superiori d'Italia sono le più ciniche di tutte le loro pari nelle altre nazioni. Il popolaccio italiano è il più cinico di tutti i popolacci» (Leopardi e Cordero, 2011: 29). In Italia il civile conversare è sostituito dall'insulto reciproco, dalla denigrazione e dall'autodenigrazione: «Le conversazioni d'Italia sono un ginnasio dove colle offensioni delle parole e dei modi s'impara per una parte e si riceve stimolo dall'altra a far male a' suoi simili co' fatti» (Leopardi e Cordero, 2011: 33). Tale violenza verbale, sintomo di deriva morale, è frutto di un'anomalia del tutto tipica della società italiana - secondo Leopardi - a raffronto con le altre nazioni mondiali: l'anomalia di una civilizzazione imperfetta. Alla cancellazione delle proprie radici culturali e delle illusioni date dai miti e dalle virtù degli antichi, non è corrisposta, in Italia, la creazione, tipica invece di altre civiltà, di un armamentario di valori generati dalla ragione e dal progresso, i quali garantiscono la convivenza civile, il senso dell'identità e l'attaccamento alla patria propri delle nazioni moderne. Manca, in Italia, quella che Leopardi definisce la «società stretta» (Leopardi e Cordero, 2011: 15ss.), vale a dire in sé coesa e legata a forti valori etici e culturali condivisi. Tale mancanza fa sì che gli italiani siano cinici e costantemente avversi gli uni agli altri, privi di ambizione e di senso morale, incapaci di cooperare, di riconoscersi in ideali comuni e dunque di giungere ad un reale progresso. II discorso, come si vede, anticipa alcuni temi che saranno sviluppati poi nella canzone La ginestra, di dodici anni successiva al Discorso ${ }^{8}$.

Quella che trapela, negli articoli sopra riportati, è non soltanto una forte dose di aggressività, ma anche una sorta di transizione metaforica (o metonimica), da parte dell'aggressore (politico o giornalista che sia), dalla forma ai contenuti. Ci si lamenta della decadenza della forma, quando invece si tratta di decadenza dei contenuti, del pensiero, degli ideali, ecc. È un po' quel che succede ai mille

8. Altri intellettuali ottocenteschi, prima e dopo il Leopardi, hanno ben inquadrato il problema dell'arretratezza civile, culturale e morale degli italiani: cf. Bollati (2011). 
catoni opinionisti della linguistica (senza essere linguisti; questi ultimi, a partire da Luca Serianni, com'è ben noto, son molto meno puristi dei non addetti ai lavori ${ }^{9}$ ) che demonizzano la televisione, i giornali, internet, gli sms, ecc., accusando tali mezzi di rovinare la buona lingua italiana piuttosto che le idee e i valori veicolati da quegli stessi mezzi. Abbiamo già visto sopra un esempio del genere (Corriere del 6 giugno 2012), nel quale si accusavano i ragazzi dei reality d'esser «gente che la grammatica non sa manco cosa sia», quando, forse, il vero bersaglio non era tanto la grammatica, in fondo non così disastrata, quanto, semmai, i contenuti dei loro discorsi.

Oppure basti un esempio come il seguente, nella sua disarmante genericità:

(8) È incredibile pensare che ancora ci sia qualcuno in lista d'attesa per un corso di calligrafia, in questi tempi cupi per la lingua italiana, quotidianamente angariata dal mordi e fuggi dei tasti degli smartphone. (repubblica.it, 29 agosto 2013)

O il vieto moralismo di un articolo come questo:

(9) LA POLITICA, in modo subdolo eveloce, è riuscita ormai a contaminare ogni cosa nel nostro modo di vivere, persino il pensiero e la lingua che utilizziamo. E non pare si tratti di una bella contaminazione. È questa la ragione per cui, per Graziella Priulla, acuta autrice del volumetto Riprendiamoci le parole, è improrogabile che si compia un drastico intervento di depurazione del lessico che, in modo più o meno consapevole, abbiamo imparato ad utilizzare, probabilmente abbagliati e certamente confusi dai significati fuorvianti ad esso arbitrariamente attribuiti. Negli ultimi decenni le nostre parole hanno subito angherie inimmaginabili e incassato colpi bassi ad opera della politica, e poiché «le parole sono come le persone: fragili e preziose» molti vocaboli della lingua italiana non possono che mostrare i lividi come esito della sofferenza e del peso delle sevizie patite. Adesso non è più lecito aspettare, è giunto il tempo di correre ai ripari avviando un periodo di serio e duro risanamento perché il linguaggio è un bene che appartiene a tutti noi, che sa gratificarci, ed è per questo che abbiamo il dovere di riappropriarcene. Il volume riporta un interessante elenco delle parole e delle espressioni che sono state maggiormente abusate, rimpinzate di accezioni inadeguate, schiaffeggiate e indegnamente mortificate in questo lungo periodo buio. (repubblica.it, 29 luglio 2012)

Varrà giusto la pena di ricordare, en passant, come, a detta dei linguisti e dati alla mano, in realtà la lingua italiana, sia scritta sia parlata, non abbia mai goduto di tanta salute (nel senso di ampia e trasversale circolazione), nella sua storia, quanto quella di oggi (cf. Rossi e Ruggiano, 2013: 11-13 e relativi riferimenti bibliografici). Non bastano certo gli emoticons, gli anglicismi e qualche abbreviazione a intaccarne il sistema. D’altra parte, il tono oracolante e accorato degli scriventi, negli articoli sopra

9. Basterebbe, tra i moltissimi riferimenti possibili, anche soltanto l'intervista di Francesco Erbani a Luca Serianni (E l'italiano? Sta bene, grazie), in repubblica.it del 20 settembre 2007. Ovviamente non è possibile ignorare l'obiettivo impoverimento degli italiani d'oggi (rispetto ad altre popolazioni mondiali) sul terreno dell'alfabetizzazione, come trapela, drammaticamente, dalla recenti indagini dell'OCSE (cf. infra, nota 14). I termini della questione, tuttavia, esulano dai compiti del presente articolo. 
citati, non fa che confermare l'incapacità dei giornalisti italiani di mantenere «la giusta distanza» (per citare il titolo di un bel film di Carlo Mazzacurati, del 2007) tra il fatto e il commento e, soprattutto, tra l'autore e la realtà da lui osservata (secondo quanto dimostrato da Loporcaro, 2005).

\section{Fatta l'Italia...: la provenienza geografica come elemento discri- minatorio}

L'aggressione e la discriminazione finora esibite assumono sembianze meno riconoscibili, ma non per questo meno pericolose, in un altro fenomeno caratteristico della nostra stampa nazionale. Mi riferisco all'abitudine di molti giornalisti di specificare spesso, con solerzia talora eccessiva, la provenienza geografica o le origini degli indiziati, anche quando questa informazione non arricchisce in alcun modo le conoscenze del lettore. Per meglio dire, aggettivi etnici quali siciliano, napoletano o calabrese sembrano accompagnare persone o cose anche in contesti negativi (reati), a differenza di etnonimi quali lombardo, milanese o torinese, limitati quasi esclusivamente a contesti neutri o positivi (successo, benessere, ecc.). Un fenomeno analogo, e decisamente più studiato, è quello che riguarda la discriminazione degli stranieri, al punto da costituire uno dei punti di forza della Carta di Roma, «il codice deontologico su migranti, richiedenti asilo, rifugiati e vittime della tratta» (Redattore Sociale, 2013: 84-85), in vigore dal 2008, nata dall'impulso dell'Alto commissariato delle Nazioni Unite per i rifugiati. La Carta di Roma chiede, tra l'altro, di «evitare di rivelare l'origine etnica o la nazionalità di migranti, richiedenti asilo o rifugiati se arrestati o colpevoli di reati, qualora tale informazione sia irrilevante ai fini della notizia» (Pistolesi, 2008: 231; sulla discriminazione etnica dell'altro cf. anche Pistolesi, 2007; Stoppiello, 1999; Orrù, 2015a, b).

Decisamente meno noto (manca, se non vado errato, bibliografia specifica in merito), ma non per questo poco frequente né meno grave, l'analogo fenomeno discriminatorio ai danni degli italiani meridionali, come risulta evidente da articoli come i seguenti (i corsivi sono miei, d'ora in avanti):

(10) Ancora un arresto per stalking in provincia di Firenze. Questa volta le manette sono scattate per un 45enne siciliano residente a Scandicci, fermato martedi scorso mentre ancora si aggirava sotto casa della donna. (repubblica.it, 30 gennaio 2014)

(11) Una ventina di villette, di proprietà della Green Life, la società che fa capo a Ciro Smiraglia (napoletano trapiantato a Roma) sono state sequestrate dalla Guardia di Finanza e dalla polizia sulle alture di Cogoleto. (repubblica.it, 4 febbraio 2014)

(12) Da sabato pomeriggio, i militari guidati dal capitano Cataldo Pantaleo hanno effettuato una decina di perquisizioni in quelli che erano i luoghi in cui l'uomo - un 50enne dall'accento meridionale - era solito trovarsi. (repubblica.it, 21 gennaio 2014) 
(13) I rapinatori uomini erano due entrambi con accento meridionale - spiega il proprietario Angelo Dalla Tor - sono entrati e uno di loro ha saltato il bancone aggredendo mia figlia. Non appena mi sono girato per aiutarla l'altro mi ha preso alle spalle. (corriere.it, 10 gennaio 2014)

Siamo davvero sicuri che il riferimento alla provenienza o all'accento dei colpevoli, o presunti tali, sia un dato essenziale, nell'economia della notizia?

La forza dello stereotipo è talmente dirompente che anche un esempio di contesto positivo, come il seguente, lascia trapelare il pregiudizio discriminatorio: tutti i meridionali, e in particolare i napoletani, sono disorganizzati e confusionari, pertanto se X è flemmatico e ha «grandi doti di organizzatore» è l'eccezione che conferma la regola («quasi un ossimoro»). Si sta parlando di Giuseppe Castagna, nuovo amministratore delegato della Banca Popolare di Milano:

(14) Nessuno se lo ricorda al centro di una scenata o mentre alza la voce - anche se qualcuno gli attribuisce una certa testardaggine - e tutti gli riconoscono grandi doti di organizzatore; quasi un ossimoro, per un napoletano che dopo una vita al Nord mantiene ancora la dolcezza della cadenza meridionale. Insomma, è un manager tranquillo e riflessivo, portato naturalmente alla mediazione anche se come molte persone miti ha grandi impuntature private, racconta chi lo conosce. (repubblica.it, 20 gennaio 2014)

Qualche altro esempio significativo:

(15) I banditi intanto non si fermano. Tornano in azione all'una del 26 giugno a Laghirano, ai danni della Montepaschi di via Fanti d'Italia. Due i rapinatori all'interno dell'istituto - sempre armati di cutter - uno dei quali, come a Parma, scavalca il bancone e si fa consegnare 12.664 euro. Il bandito. per gli investigatori, è lo stesso che ha colpito in via Langhirano, al quale la Mobile, dopo uno scambio di informazioni con i colleghi di Matera, riesce a dare finalmente un nome. Si tratta di un calabrese residente a Catania, Egidio Famoso, 46 anni. A incastrarlo è la foto di Facebook, che permette alla polizia di identificarlo con certezza. Da lui si risale ai complici. II primo a finire nella rete degli inquirenti è Emanuele Grasso, catanese classe 1988, in azione nella rapina del 26 giugno a Langhirano, proprio insieme a Famoso. Le manette raggiungono anche un terzo uomo, Vincenzo Florio, che i tabulati telefonici collocano a Parma in occasione del colpo dell'11 giugno in via Langhirano. (repubblica.it, 23 gennaio 2014)

(16) IL QUARTO UOMO - I tre sono trentenni residenti nell'alto Milanese, tra Inveruno e Magenta, e almeno uno dei tre ha importanti precedenti penali. Risultano disoccupati e sono conoscenti dei fratelli Cutrì. Avrebbero precedenti per detenzione di armi, droga e reati contro il patrimonio. In mattinata, a Napoli, i carabinieri di Varese hanno fermato un quarto uomo: si tratta di un pregiudicato di origini napoletane che da tempo abitava nel Milanese. (corriere. it, 6 febbraio 2014) 
Nel primo articolo della coppia appena citata, si cita la provenienza di due uomini su tre (guarda caso meridionali). Visto che si è identificato anche il terzo (tal Vincenzo Florio), perché non specificare anche le origini di quest'ultimo (forse perché non meridionale?), o, meglio ancora, perché non omettere questo dato, ininfluente, per tutti e tre? Analogamente, nel secondo articolo, perché dei quattro uomini sospettati di aver aiutato a fuggire il boss della 'ndrangheta Domenico Cutrì si riportano le origini soltanto di uno di loro, napoletano? E gli altri tre?

Il riferimento alle origini campane dello stalker (tanto cogente da determinare l'antonomastico il campano come ripresa anaforica), nell'articolo seguente, aggiunge davvero qualche elemento utile alla notizia?

(17) Un uomo di 34 anni, di origini campane ma da anni residente a Brescia, è stato arrestato a Mirano dai carabinieri per persecuzione nei confronti di una donna. La vittima, la sera di domenica 19 gennaio, ha allertato l'Arma dopo aver visto sotto la sua abitazione il campano che aveva denunciato, per la prima volta, lo scorso dicembre in seguito ai suoi messaggi e chiamate continue, appostamenti e colloqui il più delle volte con toni minacciosi ed offensivi. (corriere.it, 20 gennaio 2014)

Sorge spontanea la domanda: e se l'uomo avesse avuto origini, che so io, liguri, l'avrebbero scritto?

L'etnonimo coinvolto nella ripresa anaforica si ha anche nell'esempio seguente, nel quale, per ben due volte, l'imputato viene stigmatizzato come il disoccupato calabrese:

(18) GLI SPARI DEL 28 APRILE - L'attentato che ora l'imputato vorrebbe cancellare risale al 28 aprile dell'anno scorso: sono circa le 11.340 quando Preiti fa fuoco sei volte contro il cordone di sicurezza dei carabinieri davanti a Palazzo Chigi. Quattro i militari colpiti: uno, appunto il brigadiere Giangrande, 50 anni, viene ferito in modo gravissimo. E subisce una lesione alla colonna vertebrale da cui non è ancora guarito. Uno choc per la Capitale: il disoccupato calabrese entra in azione mentre è in corso al Quirinale il giuramento del governo presieduto da Enrico Letta. Più tardi, arrestato, confida al pm Antonella Nespola: «Volevo sparare ai politici», [...]. Il giudice non ha tenuto conto della nostra consulenza tecnica, nella quale si è sottolineato come Preiti fosse affetto da una forte depressione e che questa patologia avesse inciso sulla sua volontà». Invece sia per la Procura, sia per il gup il disoccupato calabrese era lucido mentre sparava. Una tesi che i difensori intendono contrastare anche in secondo grado: «Preiti - sottolineano - ha perso il lavoro, poi il legame con la moglie e il figlio: è stato investito da un disastro umano e personale». (corriere.it, 21 gennaio 2014)

Ancora una volta le evidenze numeriche sono meno significative, forse, del commento puntuale caso per caso, soprattutto perché non è semplicissimo stabilire dei criteri omogenei di ricerca. Ho tuttavia voluto provare a cimentarmi con i soliti due corpora giornalistici maggiori, www.repubblica.it e www.corriere.it, ricercando i seguenti 10 termini (alla sola forma del maschile singolare): siciliano, ca- 
labrese, napoletano, campano, meridionale, lombardo, milanese, piemontese, torinese, settentrionale. Mi sono limitato alle prime 50 occorrenze, procedendo a ritroso dal 5 febbraio 2014 per Repubblica e dall's febbraio 2014 per Corriere. Ho poi distinto le attestazioni, in base al contesto di riferimento, in «negative» (quando cioè l'aggettivo si accompagna a un colpevole o presunto tale) e (in un unico insieme) «non marcate» (per esempio il cognome Calabrese, o un vino siciliano) e «positive» (nel caso in cui si elogiasse un personaggio meridionale o settentrionale). Avverto che, per economizzare i tempi dell'indagine (complicata anche dalla natura non molto flessibile dei motori di ricerca), non ho tenuto conto del fenomeno della ripetizione (vale a dire che, qualora il testo di un articolo venisse ripetuto più volte, ho comunque conteggiato anche l'attestazione ripetuta) né di quello della mancata visualizzazione delle attestazioni multiple di una stessa forma in uno stesso articolo (vale a dire che i motori di ricerca, all'interrogazione della forma siciliano, per esempio, rispondono visualizzando il termine una sola volta anche se compare più volte nel medesimo articolo). Per tutti questi motivi, sono ben conscio dei limiti di un'indagine siffatta, della quale, tuttavia, riporto di seguito i risultati (tabelle 1-4). Ricordo che i numeri si riferiscono alle occorrenze dell'etnonimo fino a un totale di 50 occorrenze conteggiate per ciascuna delle 10 forme.

Tabella 1: Occorrenze degli etnonimi in repubblica.it. Con + si intende «contesti non marcati o positivi», con - «contesti negativi»

\begin{tabular}{|c|c|c|c|c|c|}
\hline \multicolumn{6}{|c|}{ SUD } \\
\hline & siciliano & calabrese & napoletano & campano & meridionale \\
\hline+ & 48 & 41 & 48 & 49 & 48 \\
\hline- & 2 & 9 & 2 & 1 & 2 \\
\hline \multicolumn{6}{|c|}{ NORD } \\
\hline & lombardo & milanese & piemontese & torinese & settentrionale \\
\hline+ & 50 & 48 & 50 & 48 & 50 \\
\hline- & 0 & 2 & 0 & 2 & 0 \\
\hline
\end{tabular}

Tabella 2: Suddivisione di tutte le 500 (250 per il Nord, 250 per il Sud) occorrenze degli etnonimi (in contesti positivi e negativi) in repubblica.it in base all'area geografica

\begin{tabular}{|c|c|c|}
\hline & SUD & NORD \\
\hline+ & $234(93,6 \%)$ & $246(98,4 \%)$ \\
\hline- & $16(6,4 \%)$ & $4(1,6 \%)$ \\
\hline
\end{tabular}


Tabella 3: Occorrenze degli etnonimi in corriere.it. Con + si intende «contesti non marcati o positivi», con - «contesti negativi»

\begin{tabular}{|c|c|c|c|c|c|}
\hline \multicolumn{7}{|c|}{ SUD } \\
\hline & siciliano & calabrese & napoletano & campano & meridionale \\
\hline+ & 50 & 35 & 48 & 49 & 49 \\
\hline- & 0 & 15 & 2 & 1 & 1 \\
\hline \multicolumn{7}{|c|}{ NORD } \\
\hline+ & lombardo & milanese & piemontese & torinese & settentrionale \\
\hline & 50 & 50 & 50 & 50 & 50 \\
\hline
\end{tabular}

Tabella 4: Suddivisione di tutte le 500 (250 per il Nord, 250 per il Sud) occorrenze degli etnonimi (in contesti positivi e negativi) in corriere.it in base all'area geografica

\begin{tabular}{|c|c|c|}
\hline & SUD & NORD \\
\hline+ & $231(92,4 \%)$ & $250(100 \%)$ \\
\hline- & $19(7,6 \%)$ & 0 \\
\hline
\end{tabular}

II 6,4\% delle volte che compare un etnonimo legato all'Italia meridionale nella Repubblica, esso è d'ambito negativo, mentre ciò accade soltanto l'1,6\% delle volte con gli etnonimi relativi all'Italia settentrionale. Nel Corriere i dati sono ancor più polarizzati: il 7,6\% delle volte che compare un etnonimo legato all'Italia meridionale, esso è d'ambito negativo, mentre ciò non accade mai con gli etnonimi relativi all'Italia settentrionale. Mi pare, anche e soprattutto dalla qualità degli esempi sopra commentati, che l'uso non innocente degli aggettivi etnici da parte della nostra stampa quotidiana sia evidente. Come si vede, la forma più colpita è l'etnonimo calabrese: usato negativamente il 18\% (su 50 occorrenze) delle volte da Repubblica, il 30\% dal Corriere.

Sono ben consapevole, giova ripeterlo, dei limiti di simili conteggi (tra l'altro, su numeri così esigui le percentuali non sono molto significative), che in effetti non hanno alcuna pretesa di completezza, bensì vogliono soltanto costituire un aggancio numerico (richiedente approfondimento e rilievi più puntuali su corpora estesi) per futuri spunti di riflessione. È altresì ovvio che l'uso di etnonimi non ha sempre e soltanto valore discriminatorio, ma in parte riflette la situazione effettiva di un'Italia storicamente divisa in un Nord benestante e un Sud povero, con tassi di disoccupazione a due cifre e tassi di criminalità (stando ai dati dell'ISTAT) più elevati che nel resto d'Italia. Ma questa situazione (peraltro anch'essa, come tutto, soggetta a vaglio critico) potrebbe essere riferita (con lo stesso necessario vaglio critico) anche ad alcune fasce di stranieri presenti in Italia, sulle quali, come già detto, tuttavia l'attenzione all'evitamento degli etnonimi ha suscitato e suscita ampio dibattito internazionale, invece assente, in Italia, sugli etnonimi interni. Ė anche ben probabile che la frequente specificazione dell'origine dei colpevoli o dei sospettati sia un dato presente nelle note d'agenzia stampa, pigramente riprodotto dai giornalisti. Ma, ancora una volta, andrebbero vagliati caso per caso sia l'effettiva 
utilità della precisazione (nelle agenzie come negli articoli), sia, soprattutto, il bilanciamento (tenuto conto delle proporzioni dei dati reali cui sopra si è accennato) di tali precisazioni sulla provenienza meridionale e settentrionale.

Va infine precisato che la modalità stessa della comunicazione giornalistica fa un uso preferenziale di contesti negativi (i quali «fanno più notizia») rispetto a quelli positivi. Questo farebbe dunque pensare a uno sbilanciamento verso l'uso negativo di tutti gli etnonimi. Tuttavia, dai nostri dati emerge chiaramente una prevalenza di contesti negativi associati agli etnonimi meridionali. E, per chiudere il circolo, nessuna notizia è neutra: non conta tanto dove si delinque di più, bensì come viene presentato quel dato e di quali precisazioni viene arricchito.

Siamo, con il caso degli etnonimi, sul terreno degli usi ideologici della lingua, più che sulla metariflessione linguistica. E infatti questa sezione del presente articolo mantiene tutta la sua funzione parentetica. Mi sembra altresì interessante porre il problema della discriminazione linguistica nel più ampio dibattito sulle ideologie linguistiche.

Che gli italiani non siano mai stati accoglienti gli uni con gli altri, a proposito della convivenza tra varietà regionali diverse, ce lo ha sempre dimostrato quello specchio inclemente dell'identità nazionale che è la commedia all'italiana. Innumerevoli i casi esemplari (sul Totò dialettofobo, per esempio, cf. Rossi, 2002: 64-66); bastino qui le seguenti battute tratte da La grande guerra (1959) di Mario Monicelli. Così il futuro soldato Gassman (milanese) si rivolge al soldato Sordi (romano): «Romano, eh? [...] L'italiano in fanteria, il romano in fureria». Più volte il Busacca (Gassman) ribadisce il suo razzismo antimeridionale, per esempio durante le esercitazioni belliche, rivolgendosi al sicilianissimo (di Oristano, ma sempre doppiato in siciliano, a partire dai Soliti ignoti) Tiberio Murgia, apostrofato con: «Romano! Da Parma in giù, tutti romani, e camorristi anche!». E, poco dopo, in riferimento al fatalismo di un soldato pugliese: «Quello che vi frega, a voi altri popoli non emancipati e che mangiate il sapone, è il fatalismo rinunciatario». Per arrivare, a metà film, al duetto Gassman-Sordi, che declinano entrambi l'offerta di sparare per primi al soldato austriaco: «Sei un pelandrone, sei! Altro che miope! Come tutti i romani». «Appunto. Spara tu che sei milanese.»

Scivolamento nella facile stereotipia (come a dire: «voi meridionali siete tutti uguali, ma se c'è qualcuno diverso è l'eccezione che conferma la regola») e razzismo inestirpabili, pare, nella storia e nel presente degli italiani, prontamente cristallizzati dal grande schermo (e, come abbiamo visto, abbondantemente avallati dai nostri mezzi di informazione, con minor senso critico rispetto al cinema), il quale adombra il realistico caos della varietà linguistica per poi subito fuggirne (a favore di mescidanze compromissorie con l'italiano standard, e prima fra tutte quella romanesca), «cercan[do] la rassicurazione dello stereotipo?» (Comand, 2010: 44; cf. anche Rossi, in stampa).

Peraltro la sovraesposizione filmica del romanesco continua a suscitare, anche nei mezzi di stampa, qualche insofferenza. Il giornalista Aldo Cazzullo, tra gli altri, considera troppo minoritario e discriminatorio l'uso fatto dai media visivi dei dialetti settentrionali, a tutto vantaggio del romanesco 
e di quelli meridionali. Prendendo spunto dall'ultimo film di Paolo Virzì, /l capitale umano (2013), che, contrariamente all'opinione di molti, il giornalista non considera «un film contro il Nord», bensì «un film severo sull'Italia di oggi» e un «capolavoro di uno tra i migliori registi europei», egli scrive, peraltro:

[...] l'industria cultura [sic] italiana, e in particolare la televisione e il cinema, è troppo romanocentrica, e sovente è segnata da un pregiudizio antinordista. Se senti un'attrice parlare, fuori dal set - e sempre più spesso pure sul set -, parla in romanesco. Se c'è un veneto o un piemontese in un film, è sempre un pirla. Colpi di fulmine, il cinepanettone del Natale 2012, è stato girato in Trentino, presumibilmente con i soldi del Trentino: l'unico trentino in scena recita appunto la parte del ciula. E se il Sud è raccontato ora in chiave familista e consolatoria da artisti comunque di sicuro talento come Rocco Papaleo o Checco Zalone, ora con un taglio di apparente denuncia ma in realtà di compiaciuta ammirazione per la violenza camorrista, il racconto del Nord cede facilmente allo stereotipo negativo. (Cazzullo, 2014)

\section{Un'eccezione alla regola: Slangopedia}

Passiamo ora a una vistosa eccezione a quanto visto finora, vale a dire all'utilizzazione, da parte della stampa, di argomenti metalinguistici a scopo più denigratorio che di approfondimento culturale. Si tratta del dizionario di gergalismi giovanili, in continuo arricchimento da parte degli utenti, denominato Slangopedia, amministrato da Marina Simonetti per le pagine online dell'Espresso ${ }^{10}$. Slangopedia conta, fino alla mia ultima analisi del 26 luglio 2013, 1304 voci, tutte autovalidate dagli utenti, alfabeticamente ordinate e taggate (con qualche eccezione ed errore) per provenienza geografica e ambito semantico (evidente il riferimento a Urban Dictionary ${ }^{11}$ ). Il fatto che manchi un'elaborazione scientifica dei dati inviati dagli utenti e che manchino dei criteri sociolinguistici di raccolta del materiale stesso pongono forti limiti all'operazione. Spesso, infatti, le definizioni, e ancor più le ricostruzioni etimologiche, dei singoli lemmi lasciano molto a desiderare. Tenuto conto di ciò, tuttavia, l'opera costituisce un corpus lessicale di indubbio interesse, soprattutto perché l'ambito d'uso (la lingua dei giovani italiani) rende non semplice il reperimento di materiale altrettanto ampio e diversificato (praticamente da tutte le regioni italiane, nonché dalla Svizzera). Elaborando tutti i dati presenti in Slangopedia (secondo la mia riscrittura ed elaborazione personale del lemmario, che tenta di rettificare almeno in patte gli errori del tagging del sito), si ottengono i seguenti risultati. Ecco la distribuzione areale dei 1304 lemmi (ovviamente, molti termini hanno più tags areali e tematici o sono privi di tag):

10. http://temi.repubblica.it/espresso-slangopedia/2009/05/26/cosa-ce-dentro-la-slangopedia/

11. http://www.urbandictionary.com/ 
NORD (incluso Canton Ticino): 631

Lombardia: 216

Emilia Romagna: 123

Veneto: 76

Piemonte: 70

Liguria: 69

Friuli: 47

Trentino: 12

Valle d'Aosta: 1

Canton Ticino: 17

CENTRO: 290

Lazio: 167

Toscana: 93

Umbria: 18

Marche: 12

SUD (inclusa Sardegna): 259

Campania: 83

Sicilia: 63

Puglia: 48

Sardegna: 22

Calabria: 18

Abruzzo: 15

Molise: 2

Basilicata: 8

Questa, invece, la distribuzione semantica dei temi più rappresentati:

- droga: 173; 65 termini indicano lo «spinello» o la «canna»

- $\quad$ sesso: 85

- look: 73; 18 termini indicano il «tamarro» (cioè «persona priva di gusti nel vestire, dai modi rozzi e provinciali, decisamente out socialmente, antonimo di cool, figo, fighetto, pariolino e simili») 
- scuola: 56; 34 termini indicano «marinare la scuola»

- $\quad$ alcol: 55 (attestati prevalentemente al Nord)

Dal punto di vista geografico, si conferma la maggior tendenza al gergo da parte del Nord, più volte rilevata, anche se in misura meno netta rispetto al previsto, dal momento che, contrapponendo il Nord all'insieme del Centro-Sud (Sardegna inclusa), il rapporto è 631 a 549. Sfatata definitivamente, direi, la paura pasoliniana per la morte dei dialetti, oggi grazie al cielo più vitali di qualche anno fa, tra i giovani ${ }^{12}$. Per quanto riguarda i temi, spicca lo straordinario rilievo della droga (che si sarebbe immaginato, e sperato, come ancorato più al passato che al presente) e la pressoché totale assenza di termini legati alla politica.

Naturalmente, questa aperta sulla Slangopedia non è che una piccola finestra sulle tendenze in atto nel rapporto tra lingua italiana e dialetti e anche sull'autopercezione dei parlanti (da cui l'aggancio al tema dell'ideologia linguistica) sugli usi non standard, o al limite dello standard, nell'Italia di oggi. II tema, si capisce, necessiterebbe di ben altro approfondimento, impossibile in questa sede.

\section{Conclusioni}

Certo, le conclusioni semantiche dello spoglio di Slangopedia non sono confortanti, sempre tenuto conto dell'esiguo numero dei lemmi e degli informatori e anche dello scarso vaglio critico delle fonti. Ovviamente, dopo aver puntato l'indice sulla transizione metaforica dalle forme ai contenuti, non vogliamo commettere noi lo stesso errore: è un problema (ammesso che lo sia) di contenuti (val più la droga della politica) e non di forma. Il nostro tendenziale sconforto, comunque, ci riconduce, ciclicamente, al Gramsci e al Leopardi sopra citati.

Le metariflessioni sulla lingua non sono mai fine a sé stesse, bensì rivelano sempre molto sull'ideologia delle società che le producono, come ci ha insegnato Antonio Gramsci. Se questo è vero, che interpretazione dare alle aggressioni antidialettali sopra riportate, non meno che alle esaltazioni leghistiche contro una lingua unitaria? Alle crociate giornalistiche procongiuntivo non meno che ai propositi della grammatica unica dell'italiano? E allo stantio moralismo sulla decadenza dell'italiano per colpa di internet, della televisione e dei telefoni cellulari? Il tutto a fronte dei colpi inferti dalla nostra classe politica all'insegnamento scolastico e universitario, fino al recentissimo Decreto Carrozza sulla programmazione universitaria 2013-2015, che incoraggia (seguitando i propositi di Gelmini e compagni) la creazione, in Italia, di corsi universitari interamente in lingua straniera ${ }^{13}$. Sarà davvero molto difficile, almeno per chi scrive, non abbandonarsi al pessimismo leopardiano nel considerare la società italiana odierna, non meno di quella ottocentesca, come tra le peggiori fra quelle esistenti:

12. Uno studio aggiornato sul gergo giovanile del Sud è Rati (2013), utile anche per le osservazioni sugli odierni usi del dialetto. Cf. anche, oltre al classico Radtke (1993), Fusco e Marcato (2005) e Jačová (2012).

13. Articoli 2 e 3 del Decreto: cf. http://www.istruzione.it/allegati/DM_PROGRAMMAZIONE_2013-2015_FIRMATO.pdf. 
«Certo la società che avvi in Italia - scriveva il Recanatese - è tutta di danno ai costumi e al carattere morale, senza vantaggio alcuno» (Leopardi e Cordero, 2011: 33).

In altre parole, e per concludere, la secolare questione della lingua sembra morta, oggi (almeno nei quotidiani considerati, giacché di lingua, come abbiamo visto, in rete e in contesti specialistici si continua a dibattere moltissimo), non certo perché non ci sia più nulla da dire sull'italiano (che, anzi, la crescente circolazione della nostra lingua presso parlanti stranieri, lo scollamento mai sanato tra lingua della burocrazia o delle leggi e lingua comune e gli sconfortanti risultati degli ultimi rilievi OCSE $2013^{14}$ ribadiscono, semmai, l'urgenza di un'accorta politica linguistica), bensì perché la società italiana non sembra più in grado di riflettere su sé stessa (quantomeno nei media scritti tradizionali), ovvero sul proprio primario strumento di aggregazione, cioè la lingua. Mancando una «Società stretta» (per riprendere la felice espressione leopardiana), e venuto meno anche il desiderio di costruirla, difetta anche la capacità metariflessiva, che cede dunque il passo alla mera e improduttiva aggressività fine a sé stessa o alla chiacchiera estemporanea online.

Sempre dall'assenza di una società stretta deriva, naturalmente, il bassissimo senso di appartenenza nazionale degli italiani, riflesso, oltre che dall'insofferenza per gli accenti regionali, anche dall'uso talora tendenzioso degli etnonimi da parte dei giornalisti. Non manca, certamente, chi tenta di opporsi a tale deriva. Come il seguente lettore che cosi commenta un articolo del corriere.it (del 16 gennaio 2014), e le reazioni degli altri lettori, a proposito della decisione di far avvenire nel porto di Gioia Tauro il trasbordo delle armi chimiche provenienti dalla Siria:

(19) Ho provato a scorrere i commenti ... e mi è venuto il mal di mare ... da calabrese mi sento minoranza in questo Stato che se ne è sempre sbattuto di noi (che abbiamo tutte le colpe del mondo ... ma dovremmo essere italiani sempre e non solo quando conviene a chi cerca voti, a chi deve smaltire i rifiuti tossici e nocivi delle ridenti - una volta - industrie del nord, a chi deve imporre un rigasificatore che non serve ai calabresi, a chi vuole appiopparci una centrale a carbone a Saline che non serve ai calabresi, a chi manda le navi cariche di «avanzi tossici» del ricco nord da affondare a largo delle bcoste calabresi, ecc. ecc. ecc.). Se fossimo italiani, e non razzisti o ricchi contro poveri, o istruiti contro sottoacculturati, non diremmo che visto che i Calabresi hanno la 'ndrangheta non hanno titolo ad opporsi ad una decisione simile, o che i calabresi dovrebbero opporsi ai politici corrotti ed agli ‘ndranghetisti (io lo faccio, come la gran parte dei miei conterranei, tutti i giorni rispettando le leggi e le regole del vivere civile, pagando le tasse, aiutando il mio prossimo, ecc. ecc. ...). Se fossimo italiani, un popolo, e col sale in zucca ci chiederemmo: ma perchè proprio da noi? Perchè proprio in Italia? E perchè sperimentare per la prima volta la distruzione in mare proprio qui? ... Ma preferiamo blaterare cosicchè anche la brava massaia meneghina si sorbirà il suo bel pescetto pescetto pescato

14. I dati sulle competenze di base (leggere e far di conto) di 24 paesi sono stati resi noti l'8 ottobre 2013 dall'OCSE (Organizzazione per la cooperazione e lo sviluppo economico). Per una prima discussione critica dei dati cf. http://nuovoeutile.it/litalia-vuoto-delle-competenze. 
nel mare calabro saturo e ammorbato di veleni mortali. (corriere.it, 17 gennaio 2014; non ho corretto i refusi) 


\section{Bibliografia}

Arcangeli, Massimo (2004), «Sulla proposta d'istituzione di un Consiglio Superiore della Lingua Italiana», Lingua Italiana d'Oggi (L/d'O), vol. I, Roma, Bulzoni, p. 1-8.

Aresti, Alessandro (2014), «Questioni intorno alla lingua italiana nella stampa quotidiana nazionale», in Marco Gargiulo (ed.), Lingua e cultura italiana nei mass media. Uno sguardo interdisciplinare, Roma, Aracne, p. 105-142.

Bollati, Giulio (2011), L'italiano. Il carattere nazionale come storia e come invenzione, Torino, Einaudi.

Carrafiello, Felitta (1977), «Questioni linguistiche nella stampa quotidiana italiana», in Raffaele Simone e Giulianella Ruggiero (ed.), Aspetti sociolinguistici dell'Italia contemporanea. Atti dell'VIII Congresso Internazionale di Studi [della Società di Linguistica Italiana], 2 voll., Roma, Bulzoni, p. 593-631.

Cazzullo, Aldo (2014), «ll cinema ce l'ha con il Nord», Corriere della sera, supplemento Sette, 3 febbraio 2014, disponibile su www.corriere.it.

Comand, Mariapia (2010), Commmedia all'italiana, Milano, II Castoro.

Della Valle, Valeria e Giuseppe Patota (2009), Viva il congiuntivo!, Milano, Sperling \& Kupfer.

Demartini, Silvia (2014), Grammatica e grammatiche in Italia nella prima metà del Novecento. II dibattito linguistico e la produzione testuale, Firenze, Cesati.

Fusco, Fabiana e Carla Marcato (ed.) (2005), Forme della comunicazione giovanile, Roma, il Calamo.

Gramsci, Antonio (1996), Letteratura e vita nazionale, a cura di Valentino Gerretana, $3^{a}$ edizione, Roma, Editori riuniti. [1 $1^{\text {a }}$ ed., 1975.]

Jačová, Zora (2012), «Una varietà senza storicità. Il linguaggio giovanile in Italia», Studia Romanistica, vol. 12, n², p. 31-50.

Leopardi, Giacomo e Franco Cordero (2011), Discorso sopra lo stato presente dei costumi degl'italiani seguito dai pensieri d'un italiano d'oggi, Torino, Bollati Boringhieri.

Loporcaro, Michele (2005), Cattive notizie. La retorica senza lumi dei mass media italiani, Milano, Feltrinelli.

Mordenti, Raul (1996), «Quaderni del carcere di Antonio Gramsci», in Alberto Asor Rosa (ed.), Letteratura italiana, Le opere, vol. IV (II Novecento), II (La ricerca letteraria), Torino, Einaudi, p. 553-629.

Nencioni, Giovanni (1989), «Perché non ho scritto una grammatica per la scuola», in Giovanni Nencioni, Saggi di lingua antica e moderna, Torino, Rosemberg \& Sellier, p. 222-226.

Orrù, Paolo (2015a), «Racist Discourse on social networks. A discourse analysis of Facebook posts in Italy», Rhesis. International Journal of Linguistics, Philology, and Literature, vol. 5, nº 1, p. 113-133, disponibile su www.diplist.it/rhesis. 
Orrù, Paolo (2015b), Razzismo quotidiano. La rappresentazione dello straniero nella stampa italiana (2000-2010), tesi di dottorato, Cagliari, Università degli studi di Cagliari.

Parlangeli, Oronzo (ed.) (1971), La nuova questione della lingua, Brescia, Paideia.

Pistolesi, Elena (2007), «Identità e stereotipi nel discorso conflittuale», in Elena Pistolesi e Sabine Schwarze (ed.), Vicini/lontani. Identità e alterità nella/della lingua, Frankfurt am Main, Peter Lang, p. 115-130.

Pistolesi, Elena (2008), «La banalità dell'altro: dallo stereotipo all'insulto etnico», in Stefania Taviano (ed.), Migrazione e identità culturali, Messina, Mesogea, p. 227-238.

Radtke, Edgar (1993), La lingua dei giovani, Tübingen, Niemayer.

Rati, Maria Silvia (2013), In Calabria dicono bella. Indagini sul parlato giovanile di Reggio Calabria, Roma, Società Editrice Romana.

Redattore Sociale (ed.) (2013), Parlare civile. Comunicare senza discriminare, Milano, Bruno Mondadori.

Rossi, Fabio (2002), La lingua in gioco. Da Totò a lezione di retorica, Roma, Bulzoni.

Rossi, Fabio (2011), «Gli stereotipi filmici e pubblicitari dell'italiano all'estero», in Maria Bonaria Urban et al. (ed.), Le frontiere del Sud. Culture e lingue a contatto, Cagliari, CUEC, p. 45-61.

Rossi, Fabio (in stampa), «Lingua», in Roberto De Gaetano (ed.), Lessico del cinema italiano, 3 voll., Sesto San Giovanni, Mimesis.

Rossi, Fabio e Fabio Ruggiano (2013), Scrivere in italiano. Dalla pratica alla teoria, Roma, Carocci.

Serianni, Luca (2005), «Ancora sul Consiglio Superiore della Lingua Italiana», Lingua Italiana d'Oggi (Lld'O), vol. II, Roma, Bulzoni, p. 55-66.

Sgroi, Salvatore Claudio (2010), Per una grammatica laica. Esercizi di analisi linguistica dalla parte del parlante, Torino, Utet.

Sgroi, Salvatore Claudio (2013), Dove va il congiuntivo? Ovvero il congiuntivo da nove punti di vista, Novara, UTET Università.

Stoppiello, Sabrina (1999): «Nomi e immagini dell'“altro". Un’analisi multidimensionale della stampa», Migration Studies, vol. XXXVI, nº 135, p. 417-442. 